\title{
Risk factors, Presentation, Diagnosis and Management Factors Associated with Surgery and Death in Ischemic Colitis Patients-A Retrospective Cohort Study
}

ISSN: 2637-7632

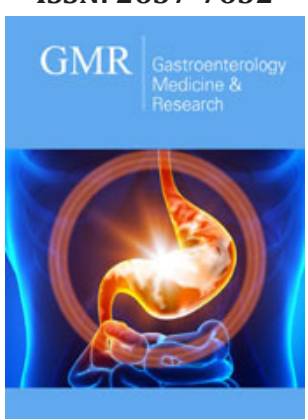

*Corresponding author: Khurram J Khan, Department of Medicine, Division of Gastroenterology, St Joseph's Healthcare Hamilton, Canada

Submission: 侮 August 19, 2019

Published: 侮August 26, 2019

Volume 3 - Issue 4

How to cite this article: Ahmad $A$ Baha A, Khurram J Khan. Risk factors, Presentation, Diagnosis and Management Factors Associated with Surgery and Death in Ischemic Colitis Patients-A Retrospective Cohort Study. Gastro Med Res. 3(4). GMR.000567. 2019 DOI: 10.31031/GMR.2019.03.000567

Copyright@ Khurram J Khan, This article is distributed under the terms of the Creative Commons Attribution 4.0 International License, which permits unrestricted use and redistribution provided that the original author and source are credited.

\author{
Ahmad Alfedaghi ${ }^{1}$, Baha Alazzoni ${ }^{1}$ and Khurram J Khan ${ }^{1,2 *}$ \\ ${ }^{1}$ Department of Medicine, Canada \\ ${ }^{2}$ Division of Gastroenterology, Canada
}

\begin{abstract}
Colonic ischemia affects older patients with co-morbidities and is usually self-limited but, in certain patients, it can be fatal. It is be suspected in any patient who presents with acute abdominal pain and bloody diarrhea and is usually diagnosed on imaging.
\end{abstract}

Methods: We searched all cases of ischemic colitis based on diagnostic codes from admissions at St Joseph's Healthcare from 2010 to 2017. The patient records were reviewed to confirm diagnosis, and clinical data collected and analyzed regarding their admission.

Results: A total of 693 patients were identified, and after exclusion, 144 were reviewed for analysis. The mean age was 70.1 years (range 27-99, SD 15), and mean length of admission was 12 days (range 0-119). Most common patient symptoms were abdominal pain (119) and hematochezia (95). Comorbidities included $73 \%$ with hypertension, 44\% with dyslipidemia, 31\% with coronary disease, and $27 \%$ with diabetes. A diagnostic CT was done in 93 patients (65\%), and a colonoscopy in 79 (55\%). Antibiotics were used in $43 \%$ of patients and blood transfusion required in $7 \%$. Mortality was $16.6 \%$ and surgery was $15.9 \%$. Complications of sepsis were $10.4 \%$ and acute kidney injury in $6.3 \%$. Ris of death was associated with sepsis $(\mathrm{p}=0.004)$, elevated INR on presentation $(\mathrm{p}=0.02)$, and history of atrial fibrillation $(\mathrm{p}=0.03)$. Risk for surgery was significantly related with sepsis $(p=0.026)$.

Conclusion: Ischemic colitis was associated with a high mortality of $16.6 \%$ and high risk of surgery of $15.9 \%$. Awareness of risk factors for poor outcome may help identify high risk patients. In our study, death was associated with patients who presented with elevated INR, past history of atrial fibrillation and those who developed sepsis.

Keywords: Ischemic colitis; Risk factors; Surgery; Mortality

\section{Introduction}

Ischemic colitis is a condition where the blood flow to part of the colon, usually the splenic flexure, is reduced causing ischemic and cellular damage to that area [1]. This can result in reversible damage, usually colitis and ulceration or irreversible damage, resulting in perforation or a stricture [2]. The cause for ischemic colitis is usually unknown most of the time but it can be secondary to systemic hypotension from any reason, low cardiac states and even following aortic surgery [2]. According to a population-based study by Hreinsson [3], 9-24\% of patients admitted to the hospital with lower gastrointestinal bleed are caused by ischemic colitis; this is only second to diverticulosis. A study of patients in the Kaiser San Diego Medical Care Program, suggested that the annual incidence for ischemic colitis was 15.6 patients in 100,000 , being more common in females above the age of 50 . The mortality rate from different studies was shown to be between $4-12 \%$ second to colonic perforation and sepsis [4].

Ischemic colitis has been associated to multiple medical and surgical conditions. Common medical risk factors include hypertension, diabetes mellitus, coronary artery disease, dyslipidemia, chronic obstructive pulmonary disease, congestive heart failure, atrial fibrillation, peripheral vascular disease and chronic kidney disease [5-7]. Patients with ischemic colitis classically present with acute abdominal pain associated with the passage fresh blood per rectum or bloody diarrhea within 24hours of symptoms. However, patients 
can present with a variety of symptoms, which ranges from nonbloody diarrhea to vomiting and syncope. This makes this condition hard to diagnose by history and examination alone. Final diagnosis is suspected on CT and confirmed with colonoscopy which will commonly shows ulceration/necrosis at the watershed area of the colon with and on biopsy there mucosal and submucosal hemorrhage and edema along with capillary fibrin thrombi and neutrophilic infiltration [8-11].

Management of ischemic colitis is dependent on the severity of the disease and mostly entails supportive measures which includes Antibiotics, intravenous fluids and blood transfusion. Antibiotics are used to prevent the translocation of bacteria from the colon; however, there is no clinical trial to describe the best antibiotic and the duration of treatment. Surgical intervention might be needed in case of colonic perforation or severe necrosis and it has been described by $0^{\prime} \mathrm{Neill}$ et al. [12]. to be necessary in $19.6 \%$ of patients with a mortality of $39.3 \%$ [12]. The objective of this study is to review patients admitted for rectal bleeding at our hospital to understand prevalence of ischemic colitis, appreciate typical presentations leading to diagnosis of ischemic colitis. We also aim to identify risk factors associated with complications, long hospital stay, surgery, and mortality as well as audit the local work-up strategy and treatment approach compared to the new international published guidelines [1].

\section{Materials and Methods}

This is a retrospective cohort study of all patients who are admitted to St Joseph's Healthcare in Hamilton, Canada under General medicine, gastroenterology, ICU and general surgery. Hamilton Integrated Research Ethics Board (HIREB) approval was obtained in October 2017 before the start of data collection. We searched all suspected cases of ischemic colitis based on diagnostic codes of admitted cases from 2010 to 2017. Information was gathered from the health records department and the keywords used for the search is ischemia/ischemic and colitis. Patients were included in the study if the diagnosis was confirmed by either CT scan or colonoscopy with histopathology. Patients were excluded if the final diagnosis is not determined, if a CT scan or colonoscopy were not done, or an alternative diagnosis is established such as ulcerative colitis, diverticulosis and mesenteric ischemia.

The cases where reviewed by the 2 authors (AA, BA) in detail, identifying all the necessary information found in the E-charts and discharge summaries over a period of 3 months on a pre-set datacollection form. EPIC was the electronicmedical health record system of St Joseph's Healthcare used to collect information regarding demographics (age, date of admission, duration of admission and symptoms at admission), medications (beta-blockers/calcium channel blockers, ACE inhibitors/ ARB, oral hyperglycemic drugs, insulin, anti-platelets/ anticoagulation, diuretics, illicit drugs and opioids), co-morbidity (hypertension, dyslipidemia, diabetes, coronary artery disease, chronic obstructive pulmonary disease/ asthma, congestive heart failure, atrial fibrillation, peripheral vascular disease, and chronic kidney disease), investigations (labs, imaging, and endoscopic procedures), management (antibiotics, blood transfusion, medications, referrals) and outcomes (discharge, surgery, complications, death).

Categorical variables were summarized by their frequency and percentage, while continuous variables were summarized by either their mean and standard deviation, if they are normally distributed, or median and inter-quartile range if they are not normally distributed. To assess the association between two categorical variables Pearson Chi-square test or Fisher's exact test were used. Moreover, the association between continuous and categorical variables was assessed using the student's t-test or nonparametric Mann-Whitney U test in case of 2 independent groups or Kruskal-Wallis one-way analysis of variance test in case of more than two groups. Logistic regression analysis was used to assess the association between a binary dependent variable. An association with a p-value of less than or equal to 0.05 was considered statistically significant. All statistical analysis was performed using SPSS Statistics for Windows Version 20.0 (Armonk, NY: IBM Corp) by one author (KJK). There was no funding for the study.

\section{Results}

Table 1: Baseline demographics.

\begin{tabular}{|c|c|}
\hline & Total Patients=144 \\
\hline Mean Age, years (SD) & $70.1(15)$ \\
\hline Mean Days of admission (range) & $64(27-99)$ \\
\hline & \\
\hline Comorbid conditions: $n$ (\%) & \\
Hypertension & $105(73)$ \\
Dyslipidemia & $64(45)$ \\
Coronary Artery Disease & $45(31)$ \\
Diabetes & $40(28)$ \\
Atrial Fibrillation & $28(19)$ \\
COPD & $26(18)$ \\
Chronic Kidney Disease & $24(16)$ \\
\hline Symptoms: $n$ (\%) & $119(83)$ \\
Abdominal pain & $95(66)$ \\
Hematochezia & $36(25)$ \\
Change in bowels & $34(24)$ \\
Vomiting & \\
\hline
\end{tabular}

A total of 693 patients were identified with potential admission for ischemic colitis. Five-hundred and forty-nine were excluded and 144 were carefully reviewed for analysis. The patient demographics and characteristics are shown in Table 1. The mean age was 70.1 years (range 27-99, SD 15), and mean length of admission was 12 days (range 0-119). The most common comorbidities included $73 \%$ with hypertension, $45 \%$ with dyslipidemia, 31\% with coronary disease, $28 \%$ with diabetes, $19 \%$ with atrial fibrillation, $18 \%$ with COPD, and 16\% with chronic kidney disease. Most common patient symptoms were abdominal pain (119), hematochezia (95), change in bowels (36), and vomiting (34). (Table 1) A diagnostic CT was done in 93 patients (65\%), and a colonoscopy in 79 (55\%). Antibiotics were used in $43 \%$ of patients and blood transfusion 
required in $7 \%$. (Table 2) The overall mortality was $16.6 \%$ and surgery was $15.9 \%$. The most common complications of sepsis were $10.4 \%$ and acute kidney injury in $6.3 \%$. Logistic regression for factors associated with death were significant for complications of sepsis $(\mathrm{p}=0.004)$, prolonged INR on presentation $(\mathrm{p}=0.02)$, and history of atrial fibrillation $(\mathrm{p}=0.03)$.

Table 2: Investigations and management of patients.

\begin{tabular}{|c|c|c|}
\hline $\begin{array}{c}\text { Investigations \& } \\
\text { Management }\end{array}$ & $\begin{array}{c}\text { Total Number of } \\
\text { Patient (n=144) }\end{array}$ & Percentage of Patients (\%) \\
\hline CT scan & 93 & $64 \%$ \\
\hline Colonoscopy & 79 & $54 \%$ \\
\hline $\begin{array}{c}\text { Blood transfusion } \\
\text { Received antibi- } \\
\text { otics }\end{array}$ & 11 & $7 \%$ \\
\hline PPI infusion & 82 & $43 \%$ \\
\hline
\end{tabular}

\section{Discussion}

There are few studies that focused on the incidence, presentations, risk facts, outcomes and mortality rates of ischemic colitis. The mean age of patients with ischemic colitis that was found in our study was 70.1 which is similar to previous studies that showed that the incidence of ischemic colitis is highest in patients above the age of 70 [11]. The mean length of stay was 12 days and ranged from 1 to 119 days. This was not mentioned in previous studies and does suggest that some patients with ischemic colitis usually do well and improve quickly while some have a complicated and long stay. The commonest 2 symptoms of ischemic colitis are abdominal pain occurring in $82 \%$ and hematochezia in $65 \%$ and that goes with previous studies that showed abdominal pain happening in around 80\% [3,13]. Rectal bleeding occurred in $84 \%$ of patients in previous studies done by Longstreth GF et al which is higher than the incidence that we are describing in our study [3]. The frequency of vomiting and diarrhea was $25 \%$ and $23 \%$, respectively. Those frequencies are similar to previous studies which showed frequency of vomiting of $30 \%$ and diarrhea occurring with frequency of $19.4 \%$ [3]. This indicates that common symptoms did not change over the years and ischemic colitis should be suspected in any patient presenting with abdominal pain and rectal bleeding.

When it comes to medical risk factors for ischemic colitis, our study had similar frequency of hypertension, diabetes, coronary artery disease, congestive heart failure, chronic kidney disease, chronic obstructive pulmonary disease and peripheral arterial disease compared to previous studies [3-5]. Moreover, dyslipidemia and atrial fibrillation were more frequent in our study as opposed to previous studies [3-5]. This might indicate that atrial fibrillation is more common as previously thought and might indicate that this diagnosis should be actively investigated. In our study the patients with suspected ischemic colitis had a CT scan of their abdomen in $64 \%$ of the cases and colonoscopy in $54 \%$ of cases. This study did not distinguish if the patients has both investigations done and might make it hard to make a generalization from this information, but it gives us perspective about how often these tests are done. The low frequency of colonoscopy may be for several reasons including patient refusal, recent completion, or the diagnosis was clear from imaging scan and complications such as perforation or abscess were ruled out. Regarding the management of ischemic colitis in St Joseph's Hospital, antibiotics were given in $43 \%$ of patients. Despite their controversy of the benefits of antibiotics in ischemic colitis, it is generally recommended treat with antibiotics to early treat sepsis that happens because of bacterial translocation. This also indicates that the diagnosis was not clear from the beginning and antibiotics were felt to be unnecessary. Or it might indicate the lack of knowledge regarding the necessity of using antibiotics in ischemic colitis. Blood transfusion was used in $7 \%$ of cases which indicate that bleeding from ischemic colitis is transient and resolves with conservative management most of the time. Proton pump inhibitors were surprisingly utilized in $5 \%$ of patients and that might be due to the fear that those patients were having an upper GI bleed early during their admission. Surgical intervention of ischemic colitis was necessary in $15.9 \%$ of patients in our study which is lower than data from previous studies where it was $19.6 \%$ [12]. The only factor that we found to be associated with higher surgical intervention need was sepsis. This lower surgical intervention rate that we are describing in our study might be due to early identification, diagnosis and medical treatment but we believe possibly that it is related to high number of patients who are poor surgical candidates. This also might explain the higher mortality in our study as opposed to previous studies; a mortality of $16.6 \%$ in this study as opposed to $4-12 \%$ in previous studies. The risk factors associated with high mortality are sepsis, atrial fibrillation and higher INR. Unfortunately, our study did not separate those with atrial fibrillation who are taking warfarin from those who are not, making it hard to interpret. Nonetheless, patients with higher INR was independently associated with higher mortality which could reflect sicker patient population that require closer monitoring. The only factor that was associated with length of stay was a history of COPD. Of patients who were admitted with ischemic colitis, $72 \%$ were discharged home which indicates that most patients with ischemic colitis do well overall and only a small number of patients will have a complicated stay [14-17].

\section{Conclusion}

Ischemic colitis was associated with mortality of $16 \%$. Awareness of risk factors for poor outcome may help identify high risk patients. In our study, death was associated with patients who presented with elevated INR, history of atrial fibrillation and those who developed sepsis. Surgical intervention need was significantly higher in patients who developed sepsis (Table 3). 
Table 3: Risk factors associated with mortality and surgery (*if $\mathrm{p}$ value significant).

\begin{tabular}{|c|c|c|}
\hline Risk Factors & $\begin{array}{c}\text { Mortality Risk } \\
\text { P-value }\end{array}$ & $\begin{array}{c}\text { Surgery Risk } \\
\text { P-value }\end{array}$ \\
\hline Hypertension & 0.219 & 0.894 \\
\hline Diabetes & 0.064 & 0.635 \\
\hline Coronary artery disease & 0.268 & 0.25 \\
\hline Dyslipidemia & 0.084 & 0.163 \\
\hline Atrial fibrillation & $0.034^{*}$ & 0.961 \\
\hline Sepsis & $0.004^{*}$ & $0.026^{*}$ \\
\hline Blood Transfusion & 0.511 & 0.818 \\
\hline High INR & $0.021^{*}$ & 0.977 \\
\hline Acute kidney injury & 0.202 & 0.755 \\
\hline
\end{tabular}

Acknowledgment: The study was presented as a poster at the American College of Gastroenterology Annual meeting in Philadelphia, USA in October 2018.

\section{References}

1. Lawrence J, Brandt ACG (2015) Clinical guideline: Epidemiology, risk factors, patterns of presentation, diagnosis, and management of colon ischemia (CI). Am J Gastroenterol 110(1): 18-44.

2. Haglund U (1987) On the pathophysiology of intestinal ischemic injury. Clinical review 153(5-6): 321-324.

3. Hreinsson JP, Gumundsson S, Kalaitzakis E (2013) Lower gastrointestinal bleeding: Incidence, etiology, and outcomes in a population-based setting. Eur J Gastroenterol Hepatol 25(1): 37-43.

4. Longstreth GF, Yao JF (2009) Epidemiology, clinical features, high-risk factors, and outcome of acute large bowel ischaemic. Clin Gastroenterol Hepatol 7(10): 1075-1080.
5. Yadav S, Dave M, Varayil JE (2015) A population-based study of incidence, risk factors, clinical spectrum, and outcomes of ischemic colitis. Clin Gastroenterol Hepatol 13(4): 731-738.

6. Chang L, Kahler KH, Sarawate C, Quimbo R, Kralstein J (2008) Assessment of potential risk factors associated with ischaemic colitis. Neurogastroenterol Motil 20: 36-42.

7. Cubiella FJ, Nunez Calvo L, Gonzalez VE (2010) Risk factors associated with the development of ischaemic colitis. World J Gastroenterol 16(36): 4564-4569.

8. Longstreth GF, Yao JF (2010) Diseases and drugs that increase risk of acute large bowel ischemia. Clin Gastroenterol Hepatol 8(1): 49-54.

9. Theodoropoulou A, Koutroubakis IE (2008) Ischemic colitis: Clinical practice in diagnosis and treatment. World J Gastroenterol 14(48): 7302-7308.

10. Glauser PM, Wermuth P, Cathomas G (2011) Ischemic colitis: Clinical presentation, localization in relation to risk factors, and long-term results 35(11): 2549-2554.

11. Longo WE, Ballantyne GH, Gusberg RJ (1992) Ischemic colitis: Patterns and prognosis. Dis Colon Rectum 35(8): 726-730.

12. O'Neill S, Yalamarthi S (2012) Systematic review of the management of ischemic colitis. Colorectal Dis 14(11): e751-e763.

13. Diaz Nieto R, Varcada M, Ogunbiyi OA (2011) Systematic review on the treatment of ischemic colitis. Colorectal Dis 13(7): 744-747.

14. Reissfelder C, Sweiti H, Antolovic D (2011) Ischemic colitis: Who will survive?. Surgery 149(4): 585-592.

15. Wiesner W, Mortele KJ, Glickman JN (2001) Pneumatosis intestinalis and portomesenteric venous gas in intestinal ischemia: Correlation of CT findings with severity of ischemia and clinical outcome. AJR Am J Roentgenol 177(6): 1319-1323.

16. Alturkistany S, Artho G, Maheshwari S (2012) Transmural colonic ischemia: Clinical features and computed tomography fi ndings. Clin Imaging 36(1): 35-40.

17. Ko GY, Ha HK, Lee HJ (1997) Usefulness of CT in patients with ischemic colitis proximal to colonic cancer. AJR Am J Roentgenol 168(4): 951-956. 